\title{
Collaborative work on evaluation of ovarian toxicity 4) Two- or four-week repeated dose study of 4-vinylcyclohexene diepoxide in female rats
}

\author{
Atsushi Ito, Naomi Mafune and Takashi Kimura \\ Drug Research Section III, Fukushima Research Laboratories, R\&D Department, Toa Eiyo Ltd., 1 Tanaka, Yuno, \\ Iizaka, Fukushima 960-0280, Japan
}

(Received December 10, 2008)

\begin{abstract}
To determine the optimal administration period for evaluation of ovarian toxicity of 4vinylcyclohexene diepoxide (VCD), VCD was intraperitoneally administered to female Sprague-Dawley rats at 0 (Control), 5, 20 and $80 \mathrm{mg} / \mathrm{kg}$ once a day for 2 or 4 weeks (2- or 4-week study). To identify small follicles, serial sections of the ovaries were stained with routine hematoxylin and eosin (HE) and proliferating cell nuclear antigen (PCNA) immunohistochemistry. In the 4-week study, decrease in small follicles was observed in the ovaries at 20 and $80 \mathrm{mg} / \mathrm{kg}$. In the 2-week study, the same change was also observed at $80 \mathrm{mg} / \mathrm{kg}$. Identification of small follicles using PCNA-stained slides was easier than that using HE-stained slides. In conclusion, histopathological findings in the ovaries are important for evaluation of female reproductive toxicity of VCD, and ovarian toxicity of VCD can be detected by administration for 2 weeks at an appropriate dose level. Furthermore, PCNA immunohistochemistry is effective for evaluation of small follicle destruction in chemical-induced ovarian toxicity.
\end{abstract}

Key words: 4-Vinylcyclohexene diepoxide, Ovarian toxicity, Rats, Histopathology, PCNA

\section{INTRODUCTION}

As part of a collaborative study on toxicity related to female fertility, we performed experiments using 4-vinylcyclohexene diepoxide (VCD), the occupational chemical known to be an ovarian toxicant. VCD is a metabolite formed by epoxidation of 4-vinylcyclohexene ( $\mathrm{VCH})$, and is used industrially as a diluent for other epoxides (IARC, 1976). It has been reported that VCD administration to female rats and mice decreased small follicles of the ovaries (Smith et al., 1990). VCD is known to be an ovarian toxicant targeting small follicles directly (Hoyer and Sipes, 1996) and the mode of action (MOA) of their ovarian toxicity is acceleration of the natural process of apoptosis in small follicles (Springer et al., 1996). This acceleration is known to be associated with activation of the Bcl-2 family (Hu et al., 2001a, 2001b) and mitogen-activated protein kinase (MAPK) family (Hu et al., 2002) in small follicles. Mayer et al. (2002) reported that administration of VCD $(80 \mathrm{mg} / \mathrm{kg} /$ day, i.p. for 30 days $)$ to female rats caused premature ovarian failure by 360 days after dosing. Haas et al. (2007) reported that VCD-treated female mice can be used to model infertility in perimen- opausal women. Chemicals that deplete ovarian follicles are of toxicological concern because the mammalian ovary contains a finite number of oocyte-containing follicles. This finite pool cannot be regenerated, and thus its depletion can result in premature ovarian failure (early menopause in women; Hooser et al., 1994; Mayer et al., 2002, 2004).

The present study was designed to determine whether a 2- or 4-week administration period can detect histopathological findings in a repeated dose toxicity study to compare potential ovarian toxicity with female reproductive function in a fertility study. In the repeated dose toxicity study, VCD was intraperitoneally administered to female Sprague-Dawley rats at 0 (Control), 5, 20 and $80 \mathrm{mg} / \mathrm{kg}$ once a day for 2 or 4 weeks. To identify small follicles, serial sections of the ovaries were stained with routine hematoxylin and eosin (HE) and proliferating cell nuclear antigen (PCNA) immunohistochemistry. PCNA immunohistochemistry is well established as a tool for evaluation of follicles in the ovaries (Muskhelishvili et al., 2005). In the female fertility study, VCD was intraperitoneally administered to female rats at dose levels of $0,5,20$ and $80 \mathrm{mg} / \mathrm{kg}$ from 2 weeks before mating to day 7 of preg-

Correspondence: Atsushi Ito (E-mail: itou.atushi@toaeiyo.co.jp) 
nancy, that is for at least 3 weeks, including mating period (Kodama et al., 2009).

\section{MATERIALS AND METHODS}

\section{Animals and husbandry}

Female Sprague-Dawley rats (Slc:SD), 5 weeks-old, were purchased from Japan SLC, Inc. (Shizuoka, Japan). The rats were individually housed in a room maintained under controlled conditions of temperature $\left(23 \pm 3^{\circ} \mathrm{C}\right)$ and relative humidity $(50 \pm 20 \%)$, with a 12 -hr light/dark cycle (7:00-19:00) in a barrier system. A standard commercial diet (FR-2, Funabashi Farms CO., Ltd., Chiba, Japan) and tap water were available ad libitum. After 1week acclimatization, the rats were allocated randomly to 4 groups ( $\mathrm{n}=10$ in each group) based on their body weights in both studies.

This study conformed with the "Guide for animal experiments in Toa Eiyo Ltd.".

\section{Chemical and administration}

The chemical structure of VCD is shown in Fig. 1. VCD was obtained from Fluka (Buchs, Switzerland). VCD was dissolved in sesame oil (Sigma-Aldrich, St. Louis, MO, USA) to a final volume of $2.5 \mathrm{ml} / \mathrm{kg}$ for each dose. Control rats received the same dose volume of sesame oil.

The study design is shown in Fig. 2. VCD was intraperitoneally administered to rats at 0 (Control), 5, 20 and $80 \mathrm{mg} / \mathrm{kg}$ once a day for 2 or 4 weeks (2- or 4 -week study). Dosage levels were selected based on the results of the previous study (Smith et al., 1990).

\section{Clinical examination}

All rats were observed daily for mortality and clinical signs, and weighed twice a week. Vaginal smears were taken daily to determine the estrous cycle length in the last week of the 2-week study and in the last 2 weeks of the 4-week study.

\section{Pathological examination}

All rats were sacrificed under anesthesia the day after 2- or 4-week administration and examined macroscopically. At sacrifice, the ovaries, vagina, uterus, adrenal glands, pituitary gland, liver and kidneys were weighed and their weights relative to the final body weight were calculated. All the tissues were fixed in 10\% neutral-buffered formalin. At trimming, the bilateral ovaries were transversally halved to observe maximum area of the ovary under a light microscope. All the tissues were embedded in paraffin, sectioned and stained with HE. To identify small follicles, PCNA immunohistochemistry was performed using serial sections stained with HE. The PCNA immunohistochemical procedure was according to Muskhelishvili et al. (2005), and used anti-PCNA antibody (clone PC10, DAKO, Glostrup, Denmark). Histopathological examination was performed under a light microscope. The follicles of the ovaries were classified into three groups: small (Type 1 to $3 \mathrm{a}$ ), medium (Type $3 \mathrm{~b}$ to $5 \mathrm{a}$ ) and large (Type $5 \mathrm{~b}$ to 8 ) according to the classification of ovarian follicles reported by Pedersen and Peters (1968). The criteria for follicular reduction are shown in Table 1.

\section{Statistical analysis}

The means and standard deviations were calculated for body weights, estrous cycle length and absolute/relative organ weights. To determine whether a difference between the control group mean and each dose group mean was significant when the variance was homogeneous in Bartlett's test (Bartlett, 1937). Each dose group mean was compared with that of the control group by Dunnett's test

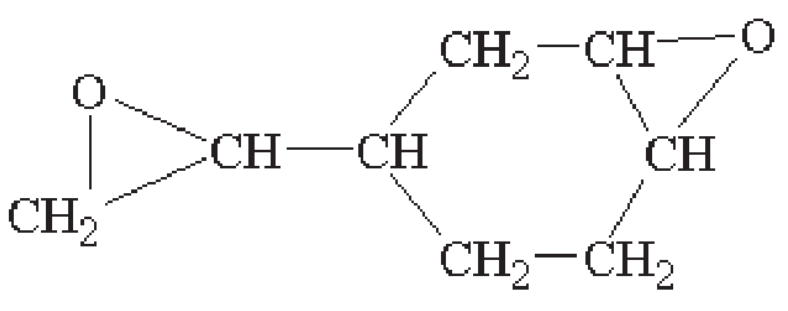

Fig. 1. Chemical structure of 4-vinylcyclohexene diepoxide.

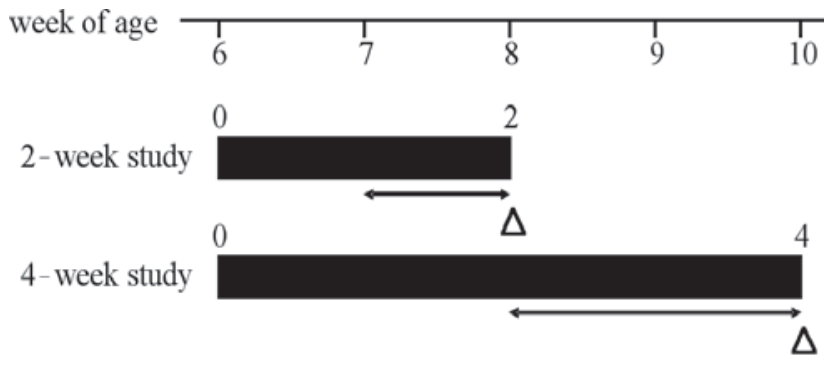

administration period $\leftrightarrow$ smear test $\Delta$ sacrifice

Dose levels: 0, 5, 20, $80 \mathrm{mg} / \mathrm{kg} / \mathrm{day}$, i.p.

Fig. 2. Study design. 
Ovarian toxicity of 4-vinylcyclohexene diepoxide in female rats

(Dunnett, 1995). When the variance was not homogeneous, Dunnett's mean rank paired comparison test was performed.

Significance levels of $5 \%$ and $1 \%$ were applied.

\section{RESULTS}

\section{Clinical examination}

There was no mortality in either study. There were no drug-related changes in clinical signs, body weight or estrous cycle length in either study.

\section{Pathological examination}

There were no abnormalities in absolute/relative organ weights or macroscopic examination except for peritonitis in either study. Peritonitis was considered to be induced by intraperitoneal injection of vehicle (sesame oil) and not to be a drug-related change since it was observed at the same level in all groups.

The results of histopathological examination of the ovaries are summarized in Table 2. Identification of small follicles using PCNA-stained slides was easier than that using HE-stained slides. Although small follicles of the ovaries were frequently observed in a control rat (Fig. 3), decrease in small follicles was observed at 20 and $80 \mathrm{mg} / \mathrm{kg}$ in the 4-week study (Fig. 4). The degree of the change at $80 \mathrm{mg} / \mathrm{kg}$ was remarkable, and few follicles were detected in 3 animals (Table 2). In the 2-week study, the same change was also observed at $80 \mathrm{mg} / \mathrm{kg}$. The degree of the change at $80 \mathrm{mg} / \mathrm{kg}$ in the 2 -week study was comparable to that at $20 \mathrm{mg} / \mathrm{kg}$ in the 4 -week study. The incidence and grade of the change were observed in a dose- and duration-dependent manner. No other histopathological findings without the reduction of small fol-

Table 1. Criteria for follicular reduction in 2- or 4-week study of 4-vinylcyclohexene diepoxide

\begin{tabular}{lccc}
\hline Finding & Grade: + & $\begin{array}{c}\text { Number of follicles } \\
\text { Grade: }++\end{array}$ & Grade: +++ \\
\hline $\begin{array}{l}\text { Unilateral ovary } \\
\text { Decrease in small follicles }\end{array}$ & $4-6$ & $2-3$ & $0-1$ \\
$\begin{array}{l}\text { Bilateral ovaries } \\
\text { Decrease in small follicles }\end{array}$ & $11-15$ & $6-10$ & $0-5$ \\
\hline
\end{tabular}

Table 2. Histopathological findings of ovaries in 2- or 4-week study of 4-vinylcyclohexene diepoxide

\begin{tabular}{|c|c|c|c|c|c|c|c|c|c|c|c|c|}
\hline \multicolumn{13}{|l|}{ 2-week study } \\
\hline Dose (mg/kg) & \multicolumn{3}{|c|}{0} & \multicolumn{3}{|c|}{5} & \multicolumn{3}{|c|}{20} & \multicolumn{3}{|c|}{80} \\
\hline No. of animals & \multicolumn{3}{|c|}{10} & \multicolumn{3}{|c|}{10} & \multicolumn{3}{|c|}{10} & \multicolumn{3}{|c|}{10} \\
\hline No. examined & $\mathrm{R}: 10$ & L: 10 & $\mathrm{~W}: 10$ & $\mathrm{R}: 10$ & $\mathrm{~L}: 10$ & $\mathrm{~W}: 10$ & $\mathrm{R}: 10$ & L:10 & $\mathrm{W}: 10$ & $\mathrm{R}: 10$ & L:10 & W: 10 \\
\hline Decrease in small follicles & 0 & 0 & 0 & 0 & 0 & 0 & 0 & 0 & 0 & 2 & 2 & 3 \\
\hline Slight & - & - & - & - & - & - & - & - & - & 1 & 0 & 1 \\
\hline Moderate & - & - & - & - & - & - & - & - & - & 0 & 1 & 2 \\
\hline Severe & - & - & - & - & - & - & - & - & - & 1 & 1 & 0 \\
\hline \multicolumn{13}{|l|}{ 4-week study } \\
\hline Dose $(\mathrm{mg} / \mathrm{kg})$ & & 0 & & & 5 & & & 20 & & & 80 & \\
\hline No. of animals & & 10 & & & 10 & & & 10 & & & 10 & \\
\hline No. examined & $\mathrm{R}: 10$ & L: 10 & $\mathrm{~W}: 10$ & $\mathrm{R}: 10$ & $\mathrm{~L}: 10$ & $\mathrm{~W}: 10$ & $\mathrm{R}: 10$ & $\mathrm{~L}: 10$ & $\mathrm{~W}: 10$ & $\mathrm{R}: 10$ & L:10 & $\mathrm{W}: 10$ \\
\hline Decrease in small follicles & 0 & 0 & 0 & 0 & 0 & 0 & 2 & 2 & 2 & 7 & 8 & 9 \\
\hline Slight & - & - & - & - & - & - & 2 & 2 & 1 & 3 & 3 & 2 \\
\hline Moderate & - & - & - & - & - & - & 0 & 0 & 1 & 1 & 4 & 4 \\
\hline Severe & - & - & - & - & - & - & 0 & 0 & 0 & 3 & 1 & 3 \\
\hline
\end{tabular}

R: right ovary, L: left ovary, W: whole body, -: no noteworthy findings 


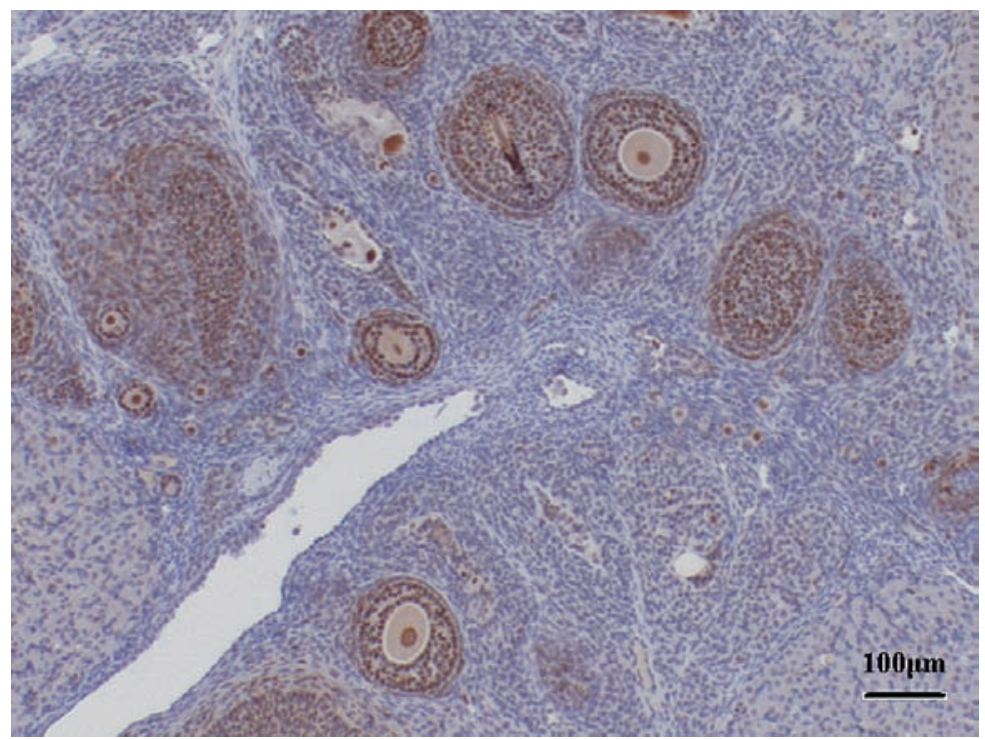

Fig. 3. In a control rat, small follicles were frequently observed. PCNA immunohistochemistry.

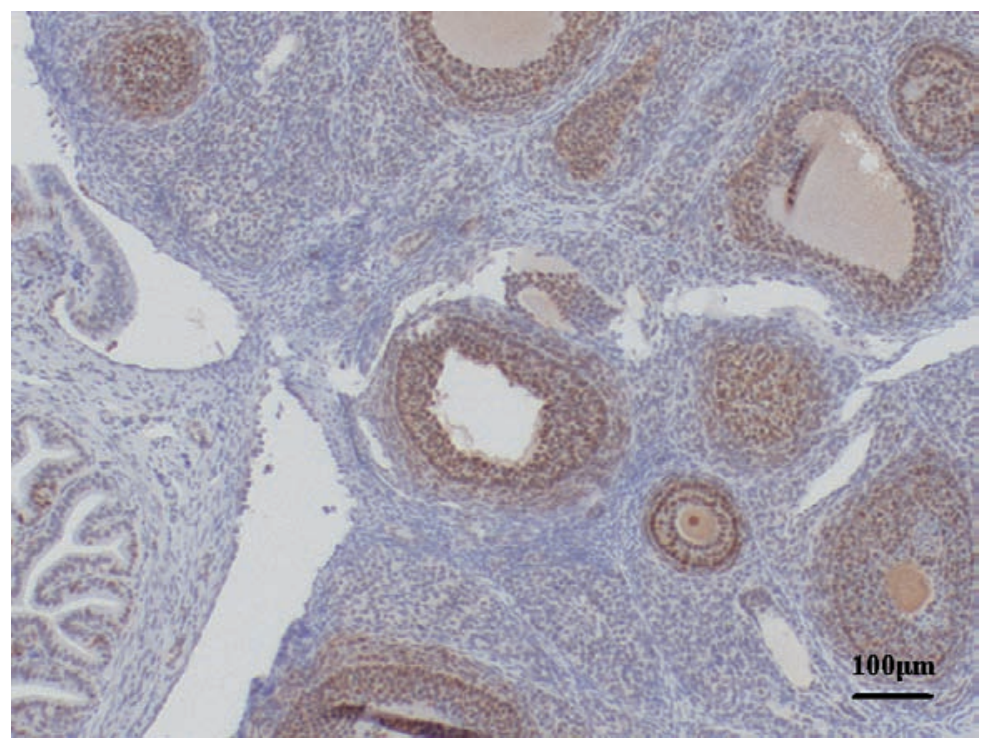

Fig. 4. Decrease in small follicles of a female rat administered with 4 -vinylcyclohexene diepoxide at $80 \mathrm{mg} / \mathrm{kg}$ for 4 weeks. PCNA immunohistochemistry.

licles were detected in the ovaries and the other organs examined.

\section{DISCUSSION}

In the present study, we conducted the 2- or 4-week repeated dose toxicity study of VCD in rats to determine the optimal administration period concerning the effects on ovarian histopathological findings. Moreover, the no observed adverse effect level (NOAEL) for the repeated dose toxicity study focused on the ovaries, and that of the female fertility study was also compared.

In the repeated dose toxicity study, there were no drugrelated changes in any examination, except for a decrease 
Ovarian toxicity of 4-vinylcyclohexene diepoxide in female rats

in small follicles of the ovaries. It has also been reported that there were no effects on the estrous cycle and organ weights (ovary, uterus, liver, kidney) in immature (30 days) rats dosed with VCD (80 mg/ $\mathrm{kg} /$ day, i.p. for 30 days) (Flaws et al., 1994). In histopathological examination, decrease in small follicles was observed at $80 \mathrm{mg} / \mathrm{kg}$ and $20 \mathrm{mg} / \mathrm{kg}$ or above in the 2- and 4-week study, respectively. Kodama et al. (2009) also reported that the same change was observed at $80 \mathrm{mg} / \mathrm{kg}$ of VCD in the female fertility study. The present data were consistent with a previous study that VCD caused a decrease of small follicles in the ovaries (Smith et al., 1990). Thus, we consider that decrease in small follicles of the ovaries induced by VCD is the most sensitive parameter for evaluating ovarian toxicity, therefore, the NOAEL for the repeated dose toxicity study focused on the ovaries was $20 \mathrm{mg} / \mathrm{kg}$ and 5 $\mathrm{mg} / \mathrm{kg}$ in the 2- and 4-week study, respectively.

A female is born with a finite number of small follicles and, once destroyed, no more can be generated.Therefore, exposure to chemicals that target small follicles can impair reproductive function and cause premature menopause in women (Hooser et al., 1994; Mayer et al., 2002, 2004). If any deviation from the normal small follicle condition is found on routine histopathological examination, it might be an indication of ovarian toxicity, and more precise histopathological examination, including PCNA immunohistochemistry, should be added. Muskhelishvili et al. (2005) reported that in rat formalin-fixed, paraffin-embedded ovary sections follicles of all degrees of maturity can be visualized by PCNA immunohistochemistry, and follicles are easily distinguished from ovarian background with the ability to detect and identify primordial follicles being enhanced. Picut et al. (2008) reported that counting of primordial and primary follicles using PCNA-stained slides is preferable to that using HE-stained slides. In the present study, identification of small follicles using PCNA-stained slides was easier than that using HEstained slides. Therefore, PCNA immunohistochemistry is effective for evaluation of small follicles destruction in chemical-induced ovarian toxicity.

In the female fertility study, decreased ovary weights, decreased the number of implanted embryos, decreased implantation rate and increased preimplantation loss rate as observed at $80 \mathrm{mg} / \mathrm{kg}$ of VCD. Therefore, the NOAELs for general toxicity in females, for the reproductive function in females and for the development of the next generation in females were considered to be $20 \mathrm{mg} / \mathrm{kg}, 20$ $\mathrm{mg} / \mathrm{kg}$ and more than $80 \mathrm{mg} / \mathrm{kg}$, respectively (Kodama et al., 2009). The histopathological findings of the ovaries in the 2-week repeated dose toxicity study were considered to be the same sensitive endpoint as female fertility parameters in the female fertility study for the detection of female reproductive toxicity of VCD.

In conclusion, histopathological findings in the ovaries are important for evaluation of female reproductive toxicity of VCD, and ovarian toxicity of VCD can be detected by administration for 2 weeks at an appropriate dose level. Furthermore, the histopathological findings in the ovaries in the repeated dose toxicity study performed at an appropriate dose level would predict alterations in female reproductive function in the female fertility study.

\section{ACKNOWLEDGMENTS}

The authors would like to thank Y. Sato, F. Yoneyama, Y. Ogino, T. Yoneda and K. Mori for technical assistance.

\section{REFERENCES}

Bartlett, M.S. (1937): Properties of sufficiency and statistical test. Proc. Roy. Soc., 160, 268-282.

Dunnett, C.W. (1995): A multiple comparison procedure for comparing several treatments with control. J. Am. Statist. Assoc., 50, 1096-1121.

Flaws, J.A., Doerr, J.K., Sipes, I.G. and Hoyer, P.B. (1994): Destruction of preantral follicles in adult rats by 4-vinyl-1-cyclohexene diepoxide. Reprod. Toxicol., 8, 509-514.

Haas, J.R., Christian, P.J. and Hoyer, P.B. (2007): Effects of impending ovarian failure induced by 4-vinylcyclohexene diepoxide on fertility in C57BL/6 female mice. Comp. Med., 57, 443-449.

Hooser, S.B., Douds, D.P., DeMerell, D.G., Hoyer, P.B. and Sipes, I.G. (1994): Long-term ovarian and gonadotropin changes in mice exposed to 4-vinylcyclohexene. Reprod. Toxicol., 8, 315323.

Hoyer, P.B. and Sipes, I.G. (1996): Assessment of follicle destruction in chemical-induced ovarian toxicity. Annu. Rev. Pharmacol. Toxicol., 36, 307-331.

Hu, X., Christian, P.J., Thompson, K.E., Sipes, I.G. and Hoyer, P.B. (2001a): Apoptosis induced in rats by 4-vinylcyclohexene diepoxide is associated with activation of the caspase cascades. Biol. Reprod., 65, 87-93.

Hu, X., Christian, P., Sipes, I.G. and Hoyer, P.B. (2001b): Expression and redistribution of cellular Bad, Bax, and $\mathrm{Bcl}-\mathrm{X}(\mathrm{L})$ protein is associated with VCD-induced ovotoxicity in rats. Biol. Reprod., 65, 1489-1495.

Hu, X., Flaws, J.A., Sipes, I.G. and Hoyer, P.B. (2002): Activation of mitogen-activated protein kinases and AP-1 transcription factor in ovotoxicity induced by 4-vinylcyclohexene diepoxide in rats. Biol. Reprod., 67, 718-724.

IARC (1976): Cadmium, nickel, some epoxides, miscellaneous industrial chemicals and general considerations on volatile anaesthetics. In IARC Monographs on the Evaluation of Carcinogenic Risk of Chemicals to Humans, Vol. 11, pp.141-145, International Agency for Research on Cancer, Lyon.

Kodama, T., Yoshida, J., Miwa, T., Hasegawa, D. and Masuyama, T. (2009): Collaborative work on evaluation of ovarian toxicity 4) Effects of fertility study of 4-vinylcyclohexene diepoxide in female rats. J. Toxicol. Sci., 34 (Special Issue I), SP59-SP63.

Mayer, L.P., Pearsall, N.A., Christian, P.J., Devine, P.J., Payne, 


\section{A. Ito et al.}

C.M., McCuskey, M.K., Marion, S.L., Sipes, I.G. and Hoyer, P.B. (2002): Long-term effects of ovarian follicular depletion in rats by 4-vinylcyclohexene diepoxide. Reprod. Toxicol., 16, 775-781.

Mayer, L.P., Devine, P.J., Dyer, C.A. and Hoyer, P.B. (2004): The follicle-deplete mouse ovary produces androgen. Biol. Reprod., 17, 130-138.

Muskhelishvili, L., Wingard, S.K. and Latendresse, J.R. (2005): Proliferating cell nuclear antigen - a marker for ovarian follicles counts. Toxicol. Pathol., 33, 365-368.

Pedersen, T. and Peters, H. (1968): Proposal for a classification of oocytes and follicles in the mouse ovary. J. Reprod. Fertil., 17, 555-557.
Picut, C.A., Swanson, C.L., Scully, K.L., Roseman, V.C., Parker, R.F. and Remick, A.K. (2008): Ovarian follicle counts using proliferating cell nuclear antigen (PCNA) and semi-automated image analysis in rats. Toxicol Pathol., 36, 674-679.

Smith, B.J., Mattison, D.R. and Sipes, I.G. (1990): The role of epoxidation in 4-vinylcyclohexene-induced ovarian toxicity. Toxicol. Appl. Pharmacol., 105, 372-381.

Springer, L.N., McAsey, M.E., Flaws, J.A., Tilly, J.L., Sipes, I.G. and Hoyer, P.B. (1996): Involvement of apoptosis in 4-vinylcyclohexene diepoxide-induced ovotoxicity in rats. Toxicol. Appl. Pharmacol., 139, 394-401. 